\title{
Comunicação pró-ambiental: estratégias informacionais, comportamentais e de framing em cartazes
}

\author{
Ligia Abreu Gomes Cruz \\ Fabio Iglesias \\ Universidade de Brasília, DF, Brasil
}

\begin{abstract}
Resumo
Usar cartazes para promover comportamentos pró-ambientais é um recurso popular e de baixo custo, mas geralmente baseado em senso comum. O objetivo desta pesquisa foi desenvolver um sistema de categorização para características persuasivas em cartazes pró-ambientais, baseado em um método misto qualitativo/quantitativo. A partir de uma amostra pública e critérios de saturação, foram selecionadas 75 peças publicitárias, identificando-se principalmente: estratégias persuasivas; tipo de ênfase sobre os impactos dos problemas ambientais; e tipo de comportamento. A maior parte dos cartazes enfatizavam consequências para a natureza, operacionalizavam comportamentos, apresentavam informações ou utilizavam framing positivo. Testes de associação mostraram que framings positivos foram associados à indicação de comportamento e ênfase na natureza. O oposto ocorreu com os framings negativos. Esses resultados são discutidos quanto às limitações na eficácia de cartazes pró-ambientais, assim como as vantagens de utilizar sistemas de categorização, como o aqui desenvolvido, para orientar pesquisa e intervenção na área.
\end{abstract}

Palavras-chave: Comunicação pró-ambiental; Estratégias persuasivas; Framing.

\section{Pro-environmental communication: informational, behavioral and framing strategies in posters}

\begin{abstract}
Posters designed to promote pro-environmental behavior are a popular, low-cost resource, though often based on common sense. The objective of this research was to develop a categorization system to identify persuasive characteristics in pro-environmental posters, with a mixed methods approach. Based on a public sample and data saturation, 75 pieces of Brazilian advertising were selected, by mainly identifying: persuasive strategies; type of emphasis regarding the impacts of environmental problems; type of recommended behavior. Results revealed that the majority of posters emphasized consequences towards nature, operationalized behaviors, showed information or used positive framing. Qui-square tests showed that positive framing was associated with indication of behavior and emphasis on nature. The opposite occurred with negative framing. Efficacy limitations in pro-environmental posters are discussed, along with the advantages of using categorization systems (such as the one developed) to guide research and intervention, are advocated.
\end{abstract}

Keywords: Pro-environmental communication; Persuasive strategies; Framing.

\section{Comunicación pro-ambiental: estrategías informacionales, comportamentales y de framing en carteles}

\section{Resumen}

Utilizar carteles para promover comportamientos pro-ambientales es un recurso popular, de bajo costo, pero generalmente basado en el sentido común. El objetivo de esta investigación fue desarrollar un sistema de clasificación para identificar las características de persuasión en carteles pro-ambientales, con un método mixto cualitativo/cuantitativo. A partir de una grande muestra publica y criterios de saturación, se seleccionaron 75 anuncios publicitarios brasileños, identificando principalmente: las estrategias de persuasión; el tipo de énfasis sobre los efectos de problemas ambientales; y el tipo de comportamiento recomendado. Los resultados revelaron que la mayoría de los carteles enfatizó consecuencias hacia la naturaleza, con indicación operacionalizada de comportamientos, mostró información o encuadre positivo utilizado. Testes del qui-cuadrado mostraron que encuadres positivos se asociaron con indicación de la conducta y el énfasis en la naturaleza. Lo contrario ocurrió con encuadres negativos. Se discuten las limitaciones de eficacia en carteles pro-ambientales, así como las ventajas de la utilización de sistemas de categorización (como lo que se desarrolló) para guiar la investigación y la intervención.

Palabras clave: Comunicación pro-ambiental; Estrategías persuasivas; Framing. 


\section{Introdução}

Expressões do tipo “seja consciente!" parecem imperativos bastante popularizados em iniciativas formais ou informais para mudança de comportamento. Tanto profissionais quanto cidadãos comuns frequentemente acreditam que a ausência de comportamentos essenciais para a promoção da saúde pública, da segurança ou da sustentabilidade, por exemplo, se explica pela "falta de consciência" (Ölander \& Thøgersen, 2014). Assim, campanhas e propagandas são orientadas pela noção de que qualquer mensagem de sensibilização, ao ser comunicada ao público, surtirá o efeito desejável. No entanto, promover mudanças por meio de mensagens persuasivas é ainda um dos grandes desafios da psicologia social aplicada e de áreas afins, tendo sua eficácia ameaçada por vários fatores.

Já na década de 1960, Chapanis (1965) indicava a necessidade de melhorar a inteligibilidade e compreensão de mensagens imperativas e declarativas. $\mathrm{O}$ autor argumentou que variados tipos de cartazes simplesmente não conseguem informar a tarefa a ser feita. Informação insuficiente, instruções difíceis de compreender, uso de linguagem distante do senso comum e outras características tornam impossível prever o tipo de ação que uma pessoa adota após ler um cartaz. Quando se pretende promover mudança de comportamento em uma grande quantidade de pessoas, garantir a eficácia da comunicação pode ser ainda mais complexo e os desafios na área permanecem em grande parte não superados (Boomsma, Pahl, \& Andrade, 2016; Klöckner, 2015).

Nolan, Schultz e Knowles (2009) apontam que as iniciativas publicitárias muitas vezes se baseiam em teorias intuitivas sobre o comportamento humano, pela falta de expertise, de recursos ou de tempo em sua elaboração. No entanto, quando utilizadas como estratégias para mudança de comportamento, as mensagens precisam de mais do que ser consideradas criativas ou agradáveis pelo público. Em complementação ao caráter intuitivo e artístico da área, uma peça de comunicação precisa se fundamentar em uma teoria empiricamente evidenciada e seguir princípios de influência social (Bator \& Cialdini, 2000). Assim, com base no desenvolvimento de um sistema de categorização, o presente estudo teve como objetivo identificar características persuasivas presentes em cartazes pró-ambientais no contexto brasileiro, disponibilizadas publicamente.

A elaboração de comunicação persuasiva eficaz é assunto estratégico em comportamentos como a reciclagem e a economia de água e energia, assim como na escolha por opções sustentáveis de moradia, de consumo e de meios de transporte (Klöckner, 2015). A promoção da sustentabilidade conta muito com esforços de instituições especializadas (p.ex., WWF, Greenpeace, S.O.S. Mata-atlântica) ou mesmo organizações que adotam o marketing verde, que investem em campanhas e meios de comunicação. Igualmente, no meio acadêmico, um corpo considerável de pesquisas em psicologia ambiental têm se dedicado à modificação de conhecimento, percepção, normas e atitudes por meio do uso de mensagens (Steg, Van Den Berg, \& De Groot, 2012).

\section{A Psicologia Social da Comunicação Pró-Ambiental}

A investigação científica da comunicação próambiental parte da caracterização das mensagens, o que permite a avaliação dos fatores de sucesso ou fracasso em sua eficácia. Aspectos básicos que definem um cartaz, como o local em que é afixado, o tipo de comportamento veiculado e o tom da mensagem, por exemplo, já derivaram as recomendações de apresentar comportamentos específicos, em local próximo à sua ocorrência e em formato polido, de forma a não ameaçar a liberdade pessoal (Ölander \& Thøgersen, 2014). O tom recriminador, por sua vez, se mostra fundamental na diferenciação entre cartazes proscritos e prescritos, que utilizam framings (enquadramentos) negativos e positivos (Winter, Cialdini, Bator, Rhoads, \& Sagarin, 1998). Nos chamados framings de valência, uma informação é apresentada sob um enfoque negativo ou positivo. Essa técnica é largamente utilizada em diversos domínios da psicologia, que envolvem o estudo dos processos de julgamento e decisão. Em seu formato clássico, as consequências de uma escolha são descritas de forma mais positiva ou negativa, de acordo com os diferentes níveis de risco associados às opções (Levin, Schneider, \& Gaeth, 1998). Por exemplo, o alto risco de não economizar água pode ser associado a um framing negativo, enquanto o comportamento próambiental correspondente pode ser apresentado sob um framing positivo.

De forma geral, é possível verificar que o framing positivo na comunicação pró-ambiental visa à manipulação da percepção sobre o impacto da ação individual na qualidade do ambiente (Sapiains, Beeton, \& Walker, 2016). Individual responses to climate change: Framing effects on pro-environmental behaviors. Journal of Applied Social Psychology, 46(8), 483-493.. Essa perspectiva tem sido investigada principalmente por meio dos conceitos de controle comportamental percebido e lócus de controle, que propõem a inclusão de incentivos e constrições à ação 
do indivíduo, bem como a percepção individual de controle, em modelos explicativos do comportamento pró-ambiental (Kollmus \& Agyeman, 2002). Tradicionalmente, essa inclusão tem sido promovida pela teoria do comportamento planejado (Fishbein \& Azjen, 2010). A variável controle comportamental percebido (avaliação individual sobre a possibilidade de realizar um comportamento) influencia a ação tanto direta, quanto indiretamente. Ela tem demonstrado melhores correlações com o comportamento próambiental e detém lugar de destaque em meta-análises da área (Bamberg \& Moser, 2007).

A percepção de controle sobre o comportamento pode ser em parte derivada da observação de características da situação. Mas, de acordo com Cleveland, Kalamas e Laroche (2005), é preciso considerar também a porção disposicional dessa percepção, determinada principalmente pelo lócus de controle de um indivíduo. O lócus de controle é tradicionalmente entendido como um fator de personalidade. Para os autores, no entanto, trata-se de um construto entre a transitoriedade situacional e o caráter disposicional, de forma que pode ser específico para cada domínio. No caso do domínio ambiental, eles defendem até a existência de um lócus de controle ambiental (environmental lócus of control, ELOC), que representa o quanto uma pessoa acredita que pode afetar resultados pró-ambientais por meio de suas ações. Um ELOC interno levaria o indivíduo a se considerar responsável pela situação ambiental e, para cada tipo de comportamento próambiental, existiria um ELOC específico, composto por múltiplas facetas: altruísmo-biosférico, ceticismo corporativo, motivação econômica e esforços individuais de reciclagem.

O framing negativo, por sua vez, pode ser relacionado a um conjunto de estratégias, interpretáveis por modelos explicativos semelhantes, sob o nome de apelos de medo e de culpa (Tannenbaum et al., 2015). Assim como na definição do framing negativo, recorrem à manipulação de respostas emocionais negativas. Mensagens em que uma pessoa experimenta remorso por não cumprir algo que deveria ter feito são chamadas de apelos de culpa. Seu objetivo é gerar empatia com outros ou com uma situação, o que, por consequência, ativará normas e regras sobre comportamentos que deveriam ocorrer. Caso os comportamentos esperados não estejam ocorrendo, surge a culpa. De acordo com Bamberg e Moser (2007), a culpa é um sentimento doloroso que aparece quando um indivíduo causa ou antecipa causar um evento aversivo. Sua importância prática está no fato de que ela ocasiona a sensação de obrigação moral de compensar o dano causado, o que pode ser utilizado para promover comportamentos. Os apelos de medo, por sua vez, veiculam mensagens que anunciam consequências negativas relacionadas a não obediência de recomendações, o que igualmente motiva a adoção do comportamento sugerido.

\section{As limitações do modelo de deficit da informação}

Para além do tom da mensagem, existem várias perspectivas sobre o conteúdo da mensagem a ser veiculado. O modelo explicativo mais antigo e popular para fundamentar campanhas pró-ambientais descreve uma causalidade linear (Kollmus \& Agyeman, 2002): conhecimento sobre o meio ambiente leva a atitudes ambientais, as quais causam mudança de comportamento. Assim, privilegia-se a informação como conteúdo das mensagens. Esse modelo, chamado de modelo de déficit de informação, parte do pressuposto de que as pessoas não mudam porque elas não sabem sobre o problema ou sobre como podem agir (Ölander \& Thøgersen, 2014, Steg, Van Den Berg, \& De Groot, 2012). A opção por disponibilizar conhecimento como forma de encorajar comportamentos é uma estratégia tradicional entre os elaboradores de peças de comunicação (Kollmus \& Agyeman, 2002; Steg, Van Den Berg \& De Groot, 2012) e é facilmente observável, pela presença de frases como "é preciso educar as pessoas".

Seja por meio de pesquisas ou pela observação cotidiana, no entanto, é possível reconhecer a ineficácia da mera apresentação de conhecimento como forma de promover mudança de comportamento. A disponibilidade de informação sobre os problemas ambientais está cada vez maior, no entanto essa realidade não resultou em mudanças comportamentais efetivas (Dolan et al., 2012). De acordo com Kaiser e Fuhrer (2003), isso ocorre porque o otimismo do senso comum com relação ao efeito da educação não é justificado, pois ainda que conhecimento componha um dos múltiplos fatores que influenciam o comportamento pró-ambiental, diversas pesquisas apontam suas limitações. Os autores reportam a relação indireta e, no máximo, moderada entre conhecimento ambiental e comportamento pró-ambiental. O conhecimento é apenas de uma influência distal, mediada por variáveis mais proximais. Como opção para viabilizar o uso do conhecimento, Kaiser e Fuhrer indicam o aumento de sua eficácia por meio da convergência de diferentes tipos de conhecimento. Informações sobre como o meio ambiente funciona e sobre os problemas ambientais compõem o conhecimento do tipo declarativo. Já o conhecimento 
procedural se refere às opções comportamentais e como atingir um objetivo pró-ambiental (p.ex., como economizar água). Além disso, uma pessoa precisa de conhecimento sobre a eficácia relativa de diferentes opções de ação, pois cada comportamento tem consequências diferentes, inclusive em termos de impacto para o meio ambiente. Acredita-se que o conhecimento social influencia no comportamento pró-ambiental, por meio do conhecimento sobre as intenções e motivos das outras pessoas, além da percepção de normas sociais.

Finalmente, em psicologia social um grande destaque é dado ao uso de mensagens baseadas em normas sociais. A teoria da conduta normativa, uma das perspectivas mais tradicionais na área, ressaltou a característica da saliência no estudo do efeito das normas sobre o comportamento (Cialdini, 2011). A conformidade a normas somente ocorre quando elas estão em foco, o que levou à utilização de mensagens normativas com o objetivo de salientar a informação normativa. A característica da saliência permite a diferenciação das mensagens normativas em tipos, com destaque para a norma injuntiva (sinalizando o comportamento moralmente aprovado) e a norma descritiva (apontando o comportamento predominante na situação), amplamente utilizadas em intervenções pró-ambientais (Cialdini, 2003).

\section{Estratégias persuasivas}

Para examinar a capacidade de mensagens próambientais em promover comportamentos correspondentes é preciso compreender que o uso de cartazes, avisos e a veiculação de argumentos em rádio e televisão se configuram como episódios persuasivos. O sucesso dessa tentativa, no entanto, depende especialmente de suas limitações. Enquanto a utilização de mensagens pró-ambientais em pesquisa recorre ao controle de possíveis erros, as iniciativas de gestores, publicitários e outros atores envolvidos na persuasão pró-ambiental parecem se basear no senso comum, raramente avaliando seus efeitos de forma sistemática. Caso uma abordagem dicotomizada da ciência seja a prática comum na elaboração peças de comunicação pró-ambiental, é possível que, ainda que muitas vezes popularizadas por sua repetição ou impacto entre o público, mensagens estejam causando efeitos indesejados pela falta de embasamento teórico. Limitações desconhecidas podem restringir a eficácia de mensagens próambientais, ou ainda, as próprias mensagens podem fortalecer ou causar os processos que se opõem à mudança de comportamento.

\section{Método}

\section{Amostra}

Visando a coletar um número maior de cartazes, utilizou-se uma ferramenta de busca pela internet. $\mathrm{O}$ uso de cartazes impressos limitaria e geraria vieses na amostragem, pelas dificuldades de acesso das organizações que os produzem, bem como a possibilidade de peças de campanhas anteriores já terem sido descartadas. O Google Imagens, portanto, possibilitou consulta pública e gratuita, por meio das palavras-chave: "propaganda meio ambiente" (maior número de resultados), "cartaz meio ambiente", "impresso meio ambiente", "OnG meio ambiente" e "marketing meio ambiente". O procedimento de coleta foi realizado durante o ano de 2014, com três ondas de levantamento. Em cada onda, a coleta era encerrada quando a busca deixava de apresentar cartazes dentro dos critérios de inclusão. Ao longo da segunda e terceira onda, a coleta foi sendo finalizada à medida que a repetição do material parecia se sobrepor à aparição de novas unidades de análise. Assim, esses critérios de saturação permitiram obter um total de 75 cartazes próambientais. A amostragem teve por critério de inclusão a seleção de cartazes que contivessem tentativas de persuadir o leitor a adotar comportamentos próambientais, elaborados por empresa privada, por OnG ou por órgão do governo. Cartazes que não foram elaborados com objetivo de impressão em série ou feitos por crianças em escolas, por exemplo, não foram considerados. Como último critério, apenas um cartaz era escolhido quando uma série de cartazes de uma mesma campanha surgia durante a coleta. $\mathrm{O}$ primeiro cartaz apresentado como resultado na ferramenta de imagens era escolhido e, caso estivesse ilegível, o próximo era selecionado.

\section{Análise de dados}

A comunicação pró-ambiental foi aqui operacionalizada como uma tentativa de persuasão, investigada quanto às suas características a partir de uma análise qualitativa. Após leitura inicial do material, foram elaboradas perguntas baseadas na literatura de psicologia ambiental e social (Klöckner, 2015), com o objetivo de orientar a criação de categorias: a) Qual foi a estratégia persuasiva utilizada?; b) O cartaz enfatiza o impacto dos problemas ambientais na própria natureza, ou no homem?; c) Existe indicação do comportamento que o leitor deve adotar?; d) Que tipo de comportamento é indicado?; e) Qual a motivação para a criação do cartaz. Cada categoria tinha a função de representar uma característica, para compor a descrição da persuasão contida nos cartazes. 
Com o auxílio das perguntas, as categorias foram inicialmente derivadas a partir do conteúdo da amostra. Utilizando-se o procedimento de classificação postergada (Kreppner, 2010), a amostra foi repetidamente observada, suas características foram comparadas umas com as outras, até que a identificação de padrões permitiu elaborar categorias de acordo com a especificidade do próprio material. Buscou-se, assim, a adequação à ênfase indutiva do método de análise qualitativo (Bauer \& Gaskell, 2002; Berg, 1995). As categorias foram esgotadas à medida que deixaram de surgir ocorrências que justificassem novas classificações, ao mesmo tempo em que as categorias existentes pareciam compreender a totalidade do conteúdo. Em seguida, foram reorganizadas de forma dedutiva, conforme modelos teóricos da psicologia social da comunicação pró-ambiental, considerandose que a qualidade da análise depende da relevância das categorias para o objetivo da pesquisa (Kreppner, 2010).

Esse processo foi balizado pelo critério de concordância entre juízes com relação a dois conjuntos de perguntas, sobre o conteúdo implícito e sobre o conteúdo explícito, que foram feitas para todos os cartazes. As perguntas sobre a estratégia persuasiva e ênfase dos cartazes objetivavam verificar o conteúdo implícito: conteúdo latente (intenção por trás do dado fisicamente observável, o significado transmitido) conforme Berg (1995); estrutura em profundidade, conteúdo detrás da superfície (Kreppner, 2010). Três pesquisadores, das áreas de psicologia ambiental, social e do consumidor, levantaram possíveis categorias por cartaz e, na ausência de unanimidade, discutiram os casos até atingir consenso, como nos procedimentos descritos por Creswell (2010). Às perguntas sobre motivação do cartaz, indicação e tipo do comportamento, foi atribuído o conteúdo explícito: manifesto (Kreppner, 2010); elementos concretos e contáveis, aparentes na superfície (Berg, 1995). Por ser passível de verificação pela leitura dos cartazes, o conteúdo explícito foi investigado por uma única pesquisadora (Kreppner, 2010).

Para a classificação do material de acordo com tipos resultantes de categoria, cada cartaz foi considerado em sua totalidade: forma, conteúdo textual e imagens. Igualmente ao processo de elaboração de categorias, a classificação do material foi realizada por meio de concordância entre juízes (para conteúdo implícito) e por única pesquisadora (para conteúdo explícito). Grande parte das categorias foi definida como mutuamente excludente, conforme a orientação de que uma categoria deve ser a representação de um evento único e não ambíguo. No entanto, cada cartaz apresentava características que permitiriam sua classificação em duas ou mais opções. Para assegurar a classificação em apenas uma categoria, a preponderância de uma das características foi definida como critério de desempate. Apenas na pergunta sobre o impacto causado pelos problemas ambientais foi incluída a categoria "ambos", pela recorrente dificuldade de consentimento entre juízes sobre a dominância de um dos tipos propostos. Assim, para a comparação da frequência "ênfase no homem" versus "ênfase na natureza", foram utilizados apenas os casos em que não houve dupla categorização. Cada cartaz foi categorizado ao mesmo tempo, quanto ao framing e à presença de conhecimento. Todos os cartazes foram analisados sob todas as categorias. Para a frequência final, organizada com objetivo exploratório e descritivo, foram utilizadas estatísticas descritivas e testes de associação. Em conjunto, os procedimentos combinaram estratégias analíticas qualitativas e quantitativas, configurando-se um método misto de pesquisa (Guest, 2013).

\section{Resultados}

Para análise dos dados foi elaborado um sistema para a categorização de cartazes pró-ambientais fundamentado na psicologia social. O sistema é composto de perguntas e possíveis classificações em resposta às perguntas, que permitem a classificação do conteúdo da mensagem persuasiva em tipos. As categorias, expostas conforme as perguntas que as orientaram, suas descrições e regras de inclusão, são descritas na Tabela 1 .

A amostra dos 75 cartazes foi também categorizada quanto à motivação para sua elaboração: homenagem a uma data, campanha de conscientização e propaganda da empresa. Como forma de caracterização da amostra, foi observada uma proporção semelhante de motivações do tipo homenagem a uma data $(\mathrm{N}=32)$ e campanha de conscientização $(\mathrm{N}=33)$. Juntas, essas motivações compuseram $87,8 \%$ dos cartazes.

Por meio do sistema foi possível verificar empiricamente as seguintes características da tentativa de persuasão presente nos cartazes: tipo de estratégia persuasiva, tipo de ênfase, presença ou ausência de indicação de comportamento e tipo de comportamento indicado. $\mathrm{Na}$ análise da ênfase sobre o impacto dos problemas ambientais, 27 cartazes foram excluídos, por não apresentar conteúdo sobre impactos ambientais ou por enfatizar igualmente as consequências para a vida humana e para o meio ambiente. A frequência de cartazes de acordo com o tipo de categoria está descrita na Tabela 2. 
TABELA 1

Perguntas, categorias e descrição das categorias

\begin{tabular}{|c|c|c|}
\hline Pergunta & Categorias & Descrição \\
\hline \multirow{7}{*}{$\begin{array}{l}\text { 1. Qual foi a estratégia persuasiva } \\
\text { utilizada? }\end{array}$} & 1.1 Informacional & Fornecer informações sobre os problemas e soluções ambientais. \\
\hline & $\begin{array}{l}\text { 1.2 Framing } \\
1.2 .1 \text { Negativo }\end{array}$ & $\begin{array}{l}\text { Apresentar consequências aversivas associadas aos problemas } \\
\text { ambientais. }\end{array}$ \\
\hline & 1.2.1.1 Amedrontamento & $\begin{array}{l}\text { Expor consequências aversivas dos problemas ambientais, com o } \\
\text { intuito de causar medo no leitor. }\end{array}$ \\
\hline & 1.2.1.2 Culpabilização & $\begin{array}{l}\text { Causar sentimento de culpa no leitor, associado a uma indicação de } \\
\text { que o mesmo não está fazendo sua parte para reverter os problemas } \\
\text { ambientais. }\end{array}$ \\
\hline & 1.2.2 Positivo & $\begin{array}{l}\text { Argumentar que o leitor pode e deve contribuir para a qualidade } \\
\text { ambiental. }\end{array}$ \\
\hline & 1.2.2 Responsabilização & $\begin{array}{l}\text { Destacar o papel do leitor na solução dos problemas ambientais, } \\
\text { associado ao argumento de que isso é uma responsabilidade } \\
\text { individual. }\end{array}$ \\
\hline & 1.2.2.2 Comparação Social & $\begin{array}{l}\text { Encorajar o leitor a seguir o exemplo de modelos que agem } \\
\text { pró-ambientalmente }\end{array}$ \\
\hline \multirow{4}{*}{$\begin{array}{l}\text { 2. O cartaz enfatiza o impacto dos } \\
\text { problemas ambientais na própria } \\
\text { natureza, ou no homem? }\end{array}$} & 2.1 Nenhuma ênfase & \\
\hline & 2.2 Ênfase na natureza & $\begin{array}{l}\text { Enfatizar as consequências negativas dos problemas ambientais } \\
\text { para o meio ambiente (e.g.: O mar está secando; a espécie X está } \\
\text { em extinção) }\end{array}$ \\
\hline & 2.3 Ênfase no homem & $\begin{array}{l}\text { Enfatizar as consequências negativas dos problemas ambientais para } \\
\text { a vida humana, a vida do leitor, ou de pessoas próximas ao mesmo } \\
\text { (e.g.: Milhões de pessoas estão morrendo de sede; Se a água do } \\
\text { planeta fosse dividida entre os habitantes, sua porção seria de apenas } \\
5 \text { litros; Que futuro você vai deixar para o seu filho?"). }\end{array}$ \\
\hline & 2.4 As duas ênfases & \\
\hline \multicolumn{3}{|l|}{$\begin{array}{l}\text { 3. Existe indicação do comportamento } \\
\text { que o leitor deve adotar? }\end{array}$} \\
\hline \multirow[t]{2}{*}{$\begin{array}{l}\text { 4. Que tipo de comportamento é } \\
\text { indicado? }\end{array}$} & 4.1 Abstrato & $\begin{array}{l}\text { Sugerir uma classe ampla de comportamentos (e.g.: Preserve; } \\
\text { Cuide; Recicle). }\end{array}$ \\
\hline & 4.2 Operacional & $\begin{array}{l}\text { Sugerir ações específicas e concretas (e.g.: Traga sua sacola } \\
\text { retornável; Feche a torneira; Filie-se a uma OnG). }\end{array}$ \\
\hline
\end{tabular}

TABELA 2

Frequência das características previstas pelo sistema de categorização

\begin{tabular}{llcc}
\hline \multirow{2}{*}{ Estratégia persuasiva } & Características & $N$ & \% do total de cartazes* \\
& Informacional & 23 & 30,7 \\
& Framing negativo - amedrontamento & 19 & 25,3 \\
& Framing negativo - culpabilização & 9 & 12,0 \\
& Framing negativo (total) & 28 & 37,3 \\
& Framing positivo - responsabilização & 35 & 46,7 \\
& Framing positivo - comparação social & 12 & 16,0 \\
& Framing positivo (total) & 47 & 62,7 \\
Ênfase sobre o impacto dos problemas ambientais & Ênfase no impacto na natureza & 40 & 53,3 \\
& Ênfase no impacto no homem & 31 & 41,3 \\
Indicação de comportamento & & 59 & 78,7 \\
Tipo de comportamento & Abstrato & 31 & 41,3 \\
& Operacional & 27 & 36,0 \\
\hline
\end{tabular}

*A quantidade de cartazes com uma característica foi comparada com o número total de cartazes,já que um mesmo cartaz poderia conter mais de uma característica, o que torna impossível comparações entre tipos. 
Testes do qui-quadrado para essas variáveis mostraram associações entre tipos de framing e indicação de comportamento, bem como entre tipos de framing e tipos de ênfase. Foi possível observar que estratégias persuasivas, quando utilizam framing negativo, foram associadas à ausência de indicação de comportamento a ser adotado pelo público alvo. Em contrapartida, o framing positivo mostrou-se associado à indicação de comportamento, $\chi^{2}(1, N=75)=8.58, p=0,003, V$ de Cramér $=0,34$. Além disso, uma associação foi verificada entre o uso de framing positivo e a ênfase no impacto dos problemas ambientais para a própria natureza. Já o framing negativo foi associado ao enfoque do impacto que os problemas ambientais podem ter no ser humano, $\chi^{2}(1, N=75)=4,81, p=0,028, V$ de Cramér $=0,32$.

\section{Discussão}

Uma das principais características que viabilizaram a descrição científica dos cartazes foi a estratégia persuasiva. A amostra indicou a existência de dois tipos dominantes de estratégia: informacional e framing. A estratégia de framing pôde ser dividida em framing negativo e positivo, subdivididos, respectivamente, em amedrontamento e culpabilização, responsabilização e comparação social. Framings do tipo positivo, especialmente por meio de responsabilização, foram os mais recorrentes. Igualmente, o fornecimento de conhecimento ambiental como estratégia foi identificado na maioria dos cartazes. Além disso, a indicação de comportamento e ênfase no impacto dos problemas ambientais na própria natureza compuseram as características predominantes nos cartazes.

De acordo com a literatura, fica claro que o conhecimento ambiental não é suficiente para promover o comportamento pró-ambiental, nem sequer um pré-requisito, já que pode ocorrer mesmo em sua ausência (Kollmus \& Agyeman, 2002). Entretanto, sua utilização como estratégia, após aprimoramento de seu conteúdo, ainda pode ser defendida. Kaiser e Fuhrer (2003) apontam que é preciso considerar o poder de predição de cada tipo de conhecimento. Conhecimento declarativo (saber sobre os problemas ambientais), por exemplo, não é considerado prerrogativa do comportamento pró-ambiental, mas é justamente o tipo de conhecimento que caracteriza a estratégia identificada no presente estudo. A convergência de diferentes tipos de conhecimento (i.e.: declarativo, procedural, de eficácia e social) é defendida pelos autores como alternativa e pode ser tomada como recomendação para a elaboração de cartazes. Além disso, apesar da maioria dos cartazes analisados não ter veiculado conhecimento, assim como as discussões sobre a duvidosa eficácia desse tipo de estratégia, é possível argumentar que a informação sobre os problemas ambientais deve ser uma recomendação, por combater a negação. Alguns estudos têm postulado a existência de uma espécie de mecanismo de defesa em que as evidências de degradação ambiental são simplesmente negadas, pela influência de emoções como o medo (Gifford, 2011). Esse fenômeno parece ser uma das principais barreiras psicológicas ao comportamento pró-ambiental na cultura brasileira (Iglesias, Caldas, \& Rabelo, 2014).

O uso de framings, por sua vez, precisa ser avaliado de acordo com a valência manipulada. A maior parte dos cartazes empregou o framing positivo, o que, de uma forma geral, encoraja a ação do leitor, enfatizando que a mudança dos problemas ambientais está em suas próprias mãos. Esse tipo de apelo se relaciona com a manipulação da percepção de controle comportamental, seja por meio de conceitos como controle comportamental percebido (De Leeuw, Valois, Ajzen, \& Schmidt, 2015) ou ELOC (Cleveland, Kalamas, \& Laroche, 2005). Por um lado, uma porção desse fenômeno pode estar fundamentada na observação de situações concretas. Uma estratégia persuasiva do tipo framing positivo poderia, então, apresentar informação que combate o viés cognitivo e implementar mudanças estruturais concomitantes à mensagem, alterando os dados oferecidos pela situação. Por outro lado, discute-se na literatura que a percepção do impacto de um comportamento também pode estar sujeita à influência das crenças gerais que um indivíduo tem sobre controle, sustentada em parte por traços de personalidade. Neste ponto, a eficácia dos cartazes que utilizam framing positivo poderia ser limitada, ou mesmo inviável.

A percepção de que o comportamento individual não tem impacto, em comparação ao nível macro do problema ambiental, funciona como uma barreira que impede a adoção de comportamentos pró-ambientais, mesmo quando há condições de realizá-lo. Essa limitação cognitiva na interpretação da realidade pode levar à ideia fatalista de que nada pode ser feito (Gifford, 2011). Por meio do conceito de ELOC, no entanto, é possível caracterizar as dimensões da percepção de controle sobre um comportamento pró-ambiental específico. Assim, pode-se ter como recomendação para elaboração de um framing positivo mais eficaz a manipulação das variáveis que sustentam um ELOC interno, em sua porção contexto-dependente, não apenas como medida de personalidade. $\mathrm{O}$ uso de responsabilização como estratégia parece promissor, inclusive, contando com a associação demonstrada nos 
resultados entre esse tipo de estratégia e a indicação de comportamento, em acordo com a recomendação de apresentação de conhecimento procedural para promover o comportamento pró-ambiental (Kaiser \& Fuhrer, 2003). Por sua vez, o uso de mensagens normativas é reconhecidamente eficaz na promoção de comportamento pró-ambiental (Cialdini, 2011). No entanto, a combinação entre essas normas também revelou a ocorrência do efeito bumerangue (consequência oposta ao objetivo) (Burchell, Rettie, \& Patel, 2013), nos casos em que a norma injuntiva perde sua ação pela saliência de uma norma descritiva que a contraria. Assim, a estratégia de comparação social na elaboração de cartazes deve considerar a relação de dependência entre normas social e seu contexto.

A despeito de qualquer limitação, é preciso ressaltar que a predominância do framing positivo nos cartazes é um dado favorável ao sucesso das tentativas persuasivas pró-ambientais e deve ser promovida. A opção oposta, o uso do framing negativo, ainda que reconhecidamente eficaz, inclui muitas ressalvas. No caso da manipulação de culpa ou medo, existe uma clara limitação: uma alternativa precisa ser apresentada em seguida ao evento aversivo e essa prática precisa orientar a elaboração dos cartazes que utilizam framing negativo. Conforme a recomendação de Witte (1998), a mensagem deve conter informação que comprove a eficácia de uma alternativa, bem como argumentos de que o indivíduo é capaz de adotar o comportamento sugerido. Se uma mensagem falhar em ressaltar tanto os meios, quanto a capacidade do indivíduo, a tentativa produz justamente o contrário de seu objetivo. A teoria do gerenciamento do terror (Greenberg \& Arndt, 2012) é uma das teorias psicológicas que pode explicar esse fenômeno. De acordo com seu modelo, a possibilidade iminente de morte, constante na vida humana, gera um potencial de ansiedade, ou terror, a ser constantemente gerenciado. $\mathrm{O}$ contato consciente e direto com o terror é aliviado por meio da negação e da crença de que a ameaça se localiza em um futuro distante. Essa defesa pode ser exacerbada, pela baixa percepção de eficácia em combater a ameaça. Contudo, apesar da obrigatoriedade em apresentar alternativas para o alívio da culpa, ou para evitar o medo, os framings negativos foram associados à ausência de indicação do comportamento a ser adotado pelo leitor.

Por fim, foi igualmente possível caracterizar a persuasão dos cartazes por meio das ênfases sobre o impacto dos problemas ambientais. Na maioria dos cartazes em que havia uma única ênfase, ocorreu a opção por enfatizar as consequências dos problemas ambientais para a própria natureza. Nota-se, sobretudo, que o framing positivo foi associado a esse tipo de ênfase, em oposição ao framing negativo, mais associado à vida humana. $\mathrm{O}$ argumento parece lógico, visto que consequências aversivas são mais diretamente relacionadas ao bem-estar do próprio indivíduo, ao passo que algumas perspectivas em psicologia ambiental argumentam que pessoas que atribuem um valor intrínseco ao meio ambiente são mais propícias a se responsabilizar e agir pró-ambientalmente (Corral, 2010). Considerando-se essa perspectiva, é possível argumentar ser contraproducente persuadir as pessoas a adotar uma visão de mundo em que o impacto dos problemas ambientais sobre o homem é ressaltado. Isso reforçaria o baixo valor atribuído ao meio ambiente, além de promover a separação entre homem e natureza, quando o objetivo final deveria ser promover uma integração de ambos. Assim, a dominância da ênfase na natureza, identificada na amostra, seria uma estratégia persuasiva adequada.

Desde os primeiros estudos para mapear as dimensões da atitude ambiental, existe uma recorrência de dois fatores nos resultados de pesquisas. Em um estudo dos mais citados na área, Thompson e Barton (1994) identificaram duas motivações ambientais: antropocêntrica e ecocêntrica. A primeira seria movida pelo impacto que a destruição ambiental pode ter na vida dos seres humanos e a segunda pelo valor intrínseco a plantas e animais. Sob essa perspectiva, não seria inadequada a persuasão que manipulasse a dimensão antropocêntrica do fenômeno, pois tanto essa quanto a dimensão ecocêntrica predizem comportamento pró-ambiental. A eficácia da estratégia, no entanto, pode depender se este é o fator de maior importância para o contexto em questão, ou seja, se existe congruência entre a ênfase apresentada (no homem ou na natureza) e a dimensão dominante no público-alvo (antropo ou ecocêntrica). Assim, uma recomendação básica ao uso da estratégia de ênfase em algum impacto ambiental seria a verificação dessa dominância entre os indivíduos que terão acesso aos cartazes, ou minimamente, a verificação do caráter dominante na cultura brasileira.

Ênfase no impacto dos problemas ambientais na própria natureza, uso de framing positivo e estratégia informacional foram as principais características encontradas na amostra de cartazes brasileiros. Sua análise permitiu o apontamento de possíveis limitações na eficácia persuasiva, principalmente quando a informação sobre o comportamento a ser adotado pelo leitor é falha. A caracterização da realidade brasileira, no entanto, só foi possível devido ao procedimento para a realização dessa análise. Considera-se que o procedimento de análise dos cartazes e o sistema de categorização dele derivado podem contribuir para o desenvolvimento da comunicação pró- 
ambiental, configurando-se como um exemplo, a ser aprimorado, de como investigar cientificamente peças de comunicação e como compreender as possíveis características a serem implementadas nas mesmas. Em especial, o uso do método misto, a combinação de estratégias indutiva e dedutiva, bem como a avaliação compartilhada entre juízes são encorajados.

No entanto, algumas limitações metodológicas precisam ser consideradas. Em primeiro lugar, é preciso ressaltar os problemas intrínsecos do método de amostragem pela busca na Internet, que não oferece garantia que a mesma amostra será selecionada em um estudo posterior, mesmo que sejam apresentadas informações como as datas nas quais a coleta foi realizada (Gosling \& Mason, 2015). Para solucionar esse problema, procurou-se conduzir a coleta até o ponto de repetição das peças de comunicação e foi ponderado o fato de que o acesso ao tipo de material ambicionado não seria possível por outros meios. No entanto, é possível que uma segunda coleta e o uso de palavras-chave diferentes gerem resultados distintos. Ainda, utilizou-se a opção de classificar um cartaz conforme sua característica predominante, para permitir análises estatísticas de associação. No entanto, é preciso reconhecer que a decisão de dominância entre características concorrentes é subjetiva e sujeita à vieses. Ainda que a concordância entre juízes possa ser utilizada para minimizar eventuais vieses, a possibilidade de classificação de um cartaz de acordo com todas as suas características, mesmo as mutuamente excludentes, deve ser uma alternativa a ser desenvolvida.

Novas formas de cruzar os dados podem ser exploradas, por exemplo, buscando a caracterização específica dos cartazes que tem por motivação promover uma empresa. Ainda, poderia ser utilizado um critério não explorado na presente pesquisa, como a caracterização de cartazes de OnGs pró-ambientais em comparação a cartazes de empresas privadas. A dificuldade na definição de apenas duas opções de categorização para a característica de ênfase sobre os impactos dos problemas ambientais (i.e.: nenhum, na natureza, no homem, ambos) deve ser investigada. É possível que alguma característica que poderia derivar novas categorias de classificação estejam por trás desse fenômeno, o que conduz a nova análise a ser realizada por juízes. Finalmente, para que o objetivo de investigação científica da comunicação pró-ambiental no Brasil seja atingido como um todo, é necessária a descrição de peças de comunicação que utilizam outros meios, como o rádio ou a televisão. Obviamente a investigação da eficácia propriamente dita dos tipos de cartazes ainda depende de estudos observacionais e experimentais que enfoquem no comportamento como variável de interesse.

A caracterização de uma amostra brasileira de cartazes pró-ambientais foi motivada pelas mesmas preocupações expostas por Nolan, Schultz e Knowles (2009): as mensagens persuasivas serão mais eficazes quando baseadas no entendimento de por que o comportamento não está ocorrendo e quando forem derivadas empiricamente. Acredita-se que as possibilidades da inspiração artística e intuitiva, já comumente aplicadas na elaboração de peças de comunicação, possam ser expandidas pela contribuição científica. $\mathrm{O}$ apontamento das limitações encontradas nas características, de acordo com a literatura, fornece elementos para que responsáveis por gestão ambiental, políticas públicas, campanhas publicitárias, educadores, dentre outros, possam refletir sobre a elaboração de mensagens pró-ambientais.

Em conjunto, os resultados oferecem uma análise científica de uma realidade comumente discutida apenas em teoria, ou com base em observações intuitivas e muitas vezes enviesadas da realidade. Essa análise pode contribuir no avanço teórico-metodológico da área, além de promover a investigação de pressupostos no contexto brasileiro. Ao mesmo tempo, permite a discussão sobre características comuns na comunicação pró-ambiental brasileira, que podem trazer limitações inerentes, já reconhecidas em literatura, e restringir a eficácia das mensagens persuasivas. De maneira destacada, a elaboração de um sistema para a categorização de cartazes pró-ambientais brasileiros representa um avanço que pode ser aprimorado e orientar pesquisa e intervenção na área.

\section{Referências}

Bamberg, S. \& Möser, G. (2007). Twenty years after Hines, Hungerford, and Tomera: a new meta-analysis of psychosocial determinants of pro-environmental behavior. Journal of Environmental Psychology, 27(1), 14-25. https:// doi.org/10.1016/j.jenvp.2006.12.002

Bator, R. \& Cialdini, R. B. (2000). The application of persuasion theory to the development of effective pro-environmental public service announcements. Journal of Social Issues, 56, 527-541. https://doi.org/10.1111/0022-4537.00182

Bauer, M. \& Gaskel, G. (2002). Pesquisa qualitativa com imagem, texto e som: um manual prático. Rio de Janeiro: Vozes. 
Berg, B. L. (1995). Qualitative research methods for the social sciences. Boston: Allyn and Bacon.

Boomsma, C., Pahl, S., \& Andrade, J. (2016). Imagining change: An integrative approach toward explaining the motivational role of mental imagery in pro-environmental behavior. Frontiers in psychology, 7, 1780. https://doi. org/10.3389/fpsyg.2016.01780

Burchell, K., Rettie, R., \& Patel, K. (2013). Marketing social norms: social marketing and the 'social norm approach'. Journal of Consumer Behaviour, 12(1), 1-9. https://doi.org/10.1002/cb.1395

Chapanis, A. (1965). "Words, words, words". Human Factors: The Journal of the Human Factors and Ergonomics Society, 7(1), 1-17. https://doi.org/10.1177/001872086500700101

Cialdini, R. B. (2003). Crafting normative messages to protect the environment. Current Directions in Psychological Science, 12(4), 105-109. https://doi.org/10.1111/1467-8721.01242

Cialdini, R. B. (2011). The focus theory of normative conduct. In P.A. Van Lange, A. W. Kruglanski, \& E. T. Higgins (Orgs.), Handbook of theories of social psychology (Vol. 2, pp. 295-312). New York: Sage.

Cleveland, M., Kalamas, M., \& Laroche, M. (2005). Shades of green: Linking environmental locus of control and proenvironmental behaviors. Journal of Consumer Marketing, 22(4), 198-212. https://doi.org/10.1108/07363760510605317

Corral, V. (2010). Psicología de la sustentabilidad: Un análisis de lo que nos hace proecológicos y prosociales. México: Editorial Trillas.

Creswell, J. W. (2010). Projeto de pesquisa: Métodos qualitativo, quantitativo e misto. Porto Alegre: Artmed.

De Leeuw, A., Valois, P., Ajzen, I., \& Schmidt, P. (2015). Using the theory of planned behavior to identify key beliefs underlying pro-environmental behavior in high-school students: Implications for educational interventions. Journal of Environmental Psychology, 42, 128-138. https://doi.org/10.1016/j.jenvp.2015.03.005

Dolan, P., Hallsworth, M., Halpern, D., King, D., Metcalfe, R., \& Vlaev, I. (2012). Influencing behaviour: The mindspace way. Journal of Economic Psychology, 33, 264-277. https://doi.org/10.1016/j.joep.2011.10.009

Gifford, R. (2011). The dragons of inaction: Psychological barriers that limit climate change mitigation and adaptation. American Psychologist, 66, 290-302. https://doi.org/10.1037/a0023566

Gosling, S. D. \& Mason, W. (2015). Internet research in psychology. Annual Review of Psychology, 66, 877-902. https:// doi.org/10.1146/annurev-psych-010814-015321

Greenberg, J. \& Arndt, J. (2012) Terror management theory. In P. A. Van Lange, A. W. Kruglanski, \& E. T. Higgins (Orgs.). Handbook of theories of social psychology (Vol. 1, pp. 398-415). London: Sage. https://doi.org/ 10.4135/9781446249215.n20

Guest, G. (2013). Describing mixed methods research: An alternative to typologies. Journal of Mixed Methods Research, 7, 141-151. https://doi.org/10.1177/1558689812461179

Iglesias, F., Caldas, L. S., \& Rabelo, L. A. T. (2014). Negando ou subestimando problemas ambientais: Barreiras psicológicas ao consumo responsável. Psico, 45(3), 377-386. https://doi.org/10.15448/1980-8623.2014.3

Kaiser, F. G., \& Fuhrer, U. (2003). Ecological behavior's dependency on different forms of knowledge. Applied Psychology, 52(4), 598-613. https://doi.org/10.1111/1464-0597.00153

Klöckner, C. (2015). The psychology of pro-environmental communication: Going beyond standard information strategies. London: Palgrave Macmillan. https://doi.org/10.1057/9781137348326

Kollmus, A. \& Agyeman, J. (2002). Mind the gap: Why do people act environmentally and what are the barriers to pro-environmental behavior? Environmental Education Research, 8(3), 239-260. https://doi.org/10.1080/ 13504620220145401

Kreppner, K. (2010). Aplicando a metodologia de observação em psicologia do desenvolvimento e da família. Curitiba: Juruá.

Levin, I. P., Schneider, S. L., \& Gaeth, G. J. (1998). All frames are not created equal: A typology and critical analysis of framing effects. Organizational Behavior and Human Decision Processes, 76(2), 149-188. https://doi.org/10.1006/ obhd.1998.2804

Nolan, J. M., Schultz, P., \& Knowles, E. S. (2009). Using public service announcements to change behavior: No more money and oil down the drain. Journal of Applied Social Psychology, 39(5), 1035-1056. https://doi.org/10.1111/ j.1559-1816.2009.00471.x

Ölander, F. \& Thøgersen, J. (2014). Informing versus nudging in environmental policy. Journal of Consumer Policy, 37(3), 341-356. https://doi.org/10.1007/s10603-014-9256-2

Sapiains, R., Beeton, R. J., \& Walker, I. A. (2016). Individual responses to climate change: Framing effects on pro-environmental behaviors. Journal of Applied Social Psychology, 46(8), 483-493. https://doi.org/10.1111/ jasp. 12378

Steg, L., Van Den Berg, A. E., \& De Groot, J. I. (Orgs.). (2012). Environmental psychology: An introduction. New York: Wiley \& Sons.

Tannenbaum, M. B., Hepler, J., Zimmerman, R. S., Saul, L., Jacobs, S., Wilson, K., \& Albarracín, D. (2015). Appealing to fear: A meta-analysis of fear appeal effectiveness and theories. Psychological Bulletin, 141(6), 1178-1204. https:// doi.org/10.1037/a0039729

Thompson, S. C. G. \& Barton, M. A. (1994). Ecocentric and anthropocentric attitudes toward the environment. Journal of Environmental Psychology, 14(2), 149-157. https://doi.org/10.1016/S0272-4944(05)80168-9 
Winter, P., Cialdini, R., Bator, R. J., Rhoads, K., \& Sagarin, B. J. (1998). An analysis of normative messages in signs at recreation settings. Journal of Interpretation Research, 3(1), 39-47.

Witte, K. (1998). Fear as motivator, fear as inhibitor: Using the extended parallel process model to explain fear appeal successes and failures. In P. A. Andersen \& L. K. Guerrero K. (Orgs.). Handbook of communication and emotion: Research, theory, applications, and contexts (pp. 423-450). San Diego: Academic Press.

\author{
Autores: \\ Ligia Abreu Gomes Cruz - Mestre, Universidade de Brasília \\ Fabio Iglesias - Doutor, Universidade de Brasília

\section{Endereço para correspondência:} \\ Ligia Abreu Gomes Cruz \\ Laboratório de Psicologia Social ICC Sul A1-116 \\ Universidade de Brasília \\ 70900-100 - Asa Norte, Brasília, DF, Brasil \\ <ligiaabreugc@gmail.com>
}

Recebido em: 20.10.2016

Aceito em: 04.10.2017 